\title{
Estrategia para la accesibilidad académica a la educación superior universitaria de una persona con discapacidad. Estudio de un caso
}

\author{
Strategy for the academic accessibility to university higher education of \\ a person with multi-disability. Study of a case
}

\section{Palabras clave}

Discapacidad, universidad, inclusión social, accesibilidad, tecnologías de apoyo, competencias.

\section{Keywords}

Disability, university, social inclusion, accessibility, assistive technology, competencies.

\section{María Andrea Guisen $<$ maguisen@gmail.com>}

Consejo Nacional de Investigaciones Científicas y Técnicas (CONICET). Argentina

\section{Introducción}

Este artículo describe y analiza una estrategia para la accesibilidad académica a la educación superior universitaria de una estudiante con discapacidad, desarrollada durante los años 2017 y 2018 mediante una metodología de tipo participativa en el marco del proyecto "Estrategias para la inclusión social de adultos con daño neurológico adquirido". En la actualidad continúa su análisis y seguimiento, asumiendo nuevos desafíos para su fortalecimiento y expansión.

La estudiante, en adelante Sol, tiene 35 años de edad. Hace 5 años, cursando el cuarto año de Psicología en la Universidad Nacional de Rosario (UNR), tuvo un accidente cerebro vascular que afectó principalmente el área motriz de su cerebro. El daño neurológico le causó hipotonía e inmovilidad de los dos miembros superiores y los dos inferiores de su cuerpo (cuadriplejía hipotónica), disminución de la agudeza visual y parálisis del aparato fonador. Sin embargo, no alteró su capacidad auditiva, su lenguaje comprensivo ni su intelecto. Sin habla o lenguaje elocutivo, su comunicación se redujo a respuestas binarias (si y no) que lograba expresar con leves gestos faciales. La disminución visual le imposibilitó leer, y el déficit

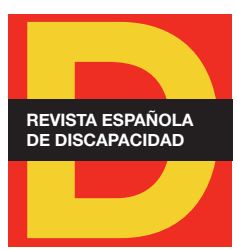

Para citar:

Guisen, M. A. (2019): "Estrategia para la accesibilidad académica a la educación superior universitaria de una persona con discapacidad. Estudio de un caso". Revista Española de Discapacidad, 7 (II): 185-200.

Doi: <https://doi.org/10.5569/23405104.07.02.11> 
motriz, escribir a mano alzada y/o tipear. Las secuelas mencionadas dificultaron su acceso a la información y a la comunicación. En esta nueva condición, Sol cesó su actividad académica en la universidad.

La UNR forma parte de la Comisión Interuniversitaria de Discapacidad y Derechos Humanos (CIDyDDHH). La misma se creó en el 2003 con la misión de bregar por la accesibilidad de los estudiantes con discapacidad a las universidades argentinas en cumplimiento de la Ley Nacional de Educación Superior $n^{\circ} 25.573$ (2002) donde se enuncia:

"El Estado, al que le cabe responsabilidad indelegable en la prestación del servicio de educación superior de carácter público (...) deberá garantizar la accesibilidad al medio físico, servicios de interpretación y los apoyos técnicos necesarios y suficientes para las personas con discapacidad" (Senado y cámara de diputados, art. 2).

En el 2007, la CIDyDDHH creó el Programa Integral de Accesibilidad en las Universidades Públicas (res. CE 426) con el fin de garantizar una educación universitaria de calidad a todos los estudiantes sin discriminación y en equidad de oportunidades. En este documento se expresan dos grandes ejes de acción: la accesibilidad física y la académica.

La física refiere de la adecuación edilicia y la disposición de equipamiento mobiliario para personas con movilidad reducida. En este aspecto en particular, la Facultad de Psicología de la UNR cuenta con un edificio accesible y con el equipamiento necesario para facilitar el ingreso y permanencia de personas con movilidad reducida.

La académica abarca, entre los aspectos más salientes: la accesibilización del material de estudio, la adaptación curricular y modalidad de cursado y evaluación, y la disposición de tecnologías de apoyo para el acceso a la información y la comunicación de los alumnos. En lo que a este eje respecta, la unidad académica se encuentra lejos de las expectativas planteadas en el programa; apenas ha avanzado en la digitalización del material de estudio.

En el año 2008, mediante la ley 26.378, Argentina ratificó la Convención sobre los Derechos de las Personas con Discapacidad (CDPD) y su protocolo facultativo (ONU, 2006)1. Si bien este instrumento jurídico excede el ámbito universitario, especialmente en el artículo 2 se abarcan tópicos vinculados a la inclusión educativa de las personas con discapacidad a los que el sistema universitario no puede ser indiferente.

En el año 2011 la CIDyDDHH presenta ante la Red de Bienestar Universitario el documento "Programa integral de accesibilidad en las universidades públicas. Profundización y avances en su implementación" (CIN, 2011). Allí expone que, 4 años después de la aprobación del Programa, no se han logrado progresos significativos en los que respecta a la accesibilidad académica, por lo que se sostiene la situación de desventaja en la que se encuentran las personas con discapacidad en las universidades. En el Acuerdo Plenario n ${ }^{\circ}$ 798/11 el Consejo Interuniversitario Nacional (CIN) avala el documento citado y le otorga carácter legal.

1. En el año 2014 a través de la ley $n^{\circ} 27.044$ se le otorga rango constitucional a la ley 26.378 ; y en el 2017 con el decreto $n^{\circ} 868 / 17$ se creó el Plan Nacional de Discapacidad (2017-2022) (Presidencia de la Nación Argentina, 2018) donde, nuevamente, se detallan cantidades de acciones a implementar que abarcan la accesibilidad universitaria de las personas con discapacidad. 
En el año 2014, cuando Sol tiene el accidente, su familia reporta la situación a la UNR quedando ésta en la lista oficial de estudiantes con discapacidad de la institución. La conformación de este listado tiene el fin de registrar aquellos alumnos que precisan de un abordaje singularizado orientado a garantizar condiciones de accesibilidad física y/o académica para su continuidad en la universidad.

Estando Sol en la lista de estudiantes con discapacidad, y vigente la estructura jurídica para la accesibilidad universitaria de las personas con discapacidad descrita en los párrafos anteriores, la UNR no implementó acciones para su continuidad.

Un año y medio más tarde, Sol comenzó a asistir al Centro de Investigación y Desarrollo en Tecnologías Especiales (CeDITE) donde ofrecen un servicio orientado a la configuración de tecnologías de apoyo para disminuir la dependencia y acrecentar la autonomía de personas con discapacidad. A comienzos del 2017, en el marco de un convenio de colaboración entre el CONICET y el CeDITE, la autora de este trabajo fue convocada para trabajar en el desarrollo de una estrategia para la inclusión social de Sol.

En los primeros encuentros con Sol también participó su madre. Al comienzo sólo mediante esta última fue posible la comunicación con Sol. Para interactuar se utilizó un sistema de comunicación de tipo físico: el abecedario escrito en un papel. La madre tocaba letra por letra, y Sol señalaba con la mirada las de su elección. La madre las recordaba y expresaba oralmente las palabras que Sol conformaba hasta componer un mensaje. Este sistema les era útil para enunciar mensajes cortos, no ideas extensas. Tampoco era transferible a la comunicación con otras personas menos cercanas, como la autora del trabajo.

En estas primeras instancias de diálogo mediado por su madre, Sol expresó: "quiero volver a la facultad para estudiar y terminar mi carrera. Puedo pensar por mí misma, pero no leer, escribir, ni hablar. No me imagino cómo podría lograrlo". La expresión de este deseo y, a su vez, la impotencia que le generaba su falta de acceso a la información y a la comunicación, fue el origen de la estrategia conjunta desarrollada para su accesibilidad académica a la educación superior universitaria.

Durante este proceso, Sol precisó realizar diferentes tareas inherentes al rol de estudiante. A su vez, para la concreción de cada una de ellas, debió rehabilitar y desarrollar diferentes competencias humanas y aprender a utilizar una cantidad de tecnologías de apoyo que se lograron elegir y configurar o desarrollar. Progresivamente, la vinculación institucional excedió la alianza original entre el CeDITE y el CONICET. Fue necesario acudir al compromiso de su familia, terapeutas, y diferentes sectores de la UNR y la Facultad de Psicología específicamente. La colaboración entre personas e instituciones fue formando una red que habilitó tanto la accesibilidad académica de Sol, como el sustento de la estrategia en el tiempo.

Objetivos y método

Dado que el marco jurídico actual que rige el funcionamiento de las universidades públicas argentinas brega por la accesibilidad de los ciudadanos a la educación superior en equidad de oportunidades, Sol debería haber gozado de condiciones óptimas para su inclusión en la carrera de Psicología de la UNR desde el momen- 
to en que su caso fue registrado en la lista de estudiantes con discapacidad. Pero ¿las leyes expresan meras promesas con el fin de paliar la lucha de los estudiantes universitarios con discapacidad y sus familiares, o promueven mecanismos para su cumplimiento? Haciendo caso omiso de la segunda opción, y ante el deseo de Sol de reincorporarse a la Facultad, surgió otra pregunta que impulsó el desarrollo de la estrategia: ¿Qué características demandaría una estrategia para la accesibilidad académica de Sol?

Durante su desarrollo emergieron nuevos interrogantes que pudieron ser dilucidados en la búsqueda de soluciones: ¿qué tareas debe realizar Sol para la regularización y aprobación de las asignaturas?, ¿qué competencias humanas y tecnologías de apoyo precisa para la realización de estas tareas? Y finalmente, ¿qué personas e instituciones intervienen en el desarrollo de una estrategia para la accesibilidad académica de Sol y su sustentabilidad en el tiempo?

Condescendiente a los interrogantes mencionados, el objetivo general de este artículo es describir y analizar la estrategia para la accesibilidad académica de Sol a la carrera de psicología de la UNR.

Para su alcance, se delimitan los siguientes objetivos específicos:

1. Reconocer las tareas que debió realizar para la regularización y aprobación de las asignaturas.

2. Relacionar estas tareas con las competencias humanas y tecnologías de apoyo implicadas en su realización.

3. Inferir las relaciones de personas e instituciones generadas en el desarrollo de la estrategia y para su sustentabilidad en el tiempo.

El objeto de análisis de este trabajo es la estrategia para la accesibilidad académica de una estudiante con discapacidad a la educación superior universitaria. Fue construido por medio de un estudio cualitativo de caso único (Stake, 1999): la estrategia para la accesibilidad académica de Sol a la facultad de Psicología de la UNR que que se dearrolló, participativamente, durante los años 2017 y 2018.

Según Stake (1999: 11) "El estudio de casos es el estudio de la particularidad y de la complejidad de un caso singular, para llegar a comprender su actividad en circunstancias importantes". Desde esta perspectiva, el abordaje de este caso de estudio se centró en: (1) conocer a Sol y sus circunstancias de vida como así también (2) el contexto universitario donde se orientó su inclusión, para luego (3) comprender el proceso a partir del cual se logró su accesibilidad académica.

(1) Para conocer a Sol y sus circunstancias de vida, se realizaron:

- Anamnesis sobre la condición psicofísica de Sol. Certificado Único de Discapacidad donde figura su diagnóstico clínico y los resultados del índice de Barthel, también conocido como "índice de discapacidad de Maryland", sobre su grado de dependencia en la realización de tareas de la vida diaria.

- Entrevista a su madre, en la que se recabó información sobre la historia de vida de la estudiante y su circunstancia (emocional, afectiva y material) actual.

- Entrevistas a sus terapeutas: acompañante terapéutica, psicóloga, kinesióloga, fonoaudióloga y oftalmólogo, en las que se recabó información respecto a los procesos de habilitación y rehabilitación de competencias de la estudiante, y sus posibilidades y limitaciones a ese momento. En estas entrevistas Sol estuvo presente y participó. 
Se registraron las entrevistas mediante grabaciones digitales. Luego se analizó el material para distinguir los aspectos más salientes a tener en cuenta en el desarrollo de la estrategia.

(2) Para conocer el contexto universitario donde se orientó su inclusión, se accedió y analizó detenidamente:

- Leyes, programas y resoluciones en donde se enuncian los derechos de los estudiantes argentinos de las universidades parte del CID y DDHH.

- $\quad$ El plan de estudio de la carrera.

Además, se mantuvieron reuniones con algunas de las autoridades de la carrera de Psicología de la UNR, con quienes se discutieron pautas de accesibilidad académica adecuadas a las singularidades de Sol, y el modo en que se la acompañaría en el transcurso hacia su inclusión.

(3) Para comprender el proceso a partir del cual se logró su accesibilidad académica:

Sol y la autora de este trabajo se encontraron con una frecuencia semanal para trabajar en el desarrollo de la estrategia. En estas instancias presenciales resolvían dudas y obstáculos, y planteaban nuevos objetivos a alcanzar. Realizaban los primeros pasos juntas y luego Sol, con el apoyo de una acompañante terapéutica y su madre, continuaba el proceso a distancia hasta el próximo encuentro. Durante estas instancias se pudieron identificar las tareas a realizar, y las competencias humanas y tecnologías de apoyo implicadas.

Se registró mediante auto grabaciones digitales y en un diario de campo lo sucedido en cada encuentro. El análisis de la información obtenida fue central para la toma decisiones en el desarrollo de la estrategia, y para comprender el proceso de accesibilidad académica en su totalidad.

Para el análisis de las competencias humanas implicadas en las tareas, se utilizaron como referencia las categorías que Creática Fundación FREE Iberoamericana para la Cooperación (2018) propone en el sitio wikinclusion.org bajo el título "Competencias para la vida". Luego se agruparon en meta categorías de elaboración propia: competencias digitales, cognitivas y emocionales.

Elegir, y configurar o desarrollar las tecnologías de apoyo empleadas implicó un largo proceso de ensayo, prueba y error. Se priorizó el uso de software de libre acceso y la realización de adaptaciones de periféricos de bajo costo. Ante la necesidad de financiamiento económico para cubrir el valor de software privativos y una notebook, se acudió a las alianzas institucionales logradas en el marco de la estrategia.

\section{Resultados y avances de la estrategia}

Los resultados y avances en el desarrollo de la estrategia se presentan en las siguientes tablas y figuras.

Las tareas, competencias y su relación configuran una gran cantidad de datos que, mediante las tablas, se proponen condensar, ordenar y sistematizar a fin de facilitar la comprensión del lector. En la tabla 1 se relacionan las tareas que Sol debió realizar en su rol de estudiante con las competencias humanas implicadas. 
En la tabla 2 se especifican cuáles de estas competencias tenía adquiridas antes de comenzar la estrategia, cuáles precisó rehabilitar y cuáles desarrollar por primera vez.

\begin{tabular}{|c|c|c|c|c|c|c|c|c|}
\hline \multicolumn{9}{|c|}{ Tabla 1. Relación entre tareas y competencias } \\
\hline \multicolumn{2}{|c|}{ Competencias } & $\begin{array}{l}1= \\
\text { interpretar } y\end{array}$ & & $\begin{array}{c}3=\text { marcar, } \\
\text { copiar }\end{array}$ & $4=$ & $\begin{array}{c}5= \\
\text { intercambiar }\end{array}$ & $\begin{array}{c}6= \\
\text { emular }\end{array}$ & $7=$ \\
\hline \multirow{4}{*}{ Digitales } & $\begin{array}{l}\text { 1= movimiento } \\
\text { preciso para } \\
\text { el uso de } \\
\text { periféricos }\end{array}$ & & & & & & & $x$ \\
\hline & $\begin{array}{l}2=\text { Uso de } \\
\text { comunicadores }\end{array}$ & & & & & & $x$ & \\
\hline & $\begin{array}{l}3=\text { Uso de } \\
\text { software de } \\
\text { ofimática }\end{array}$ & & $X$ & $x$ & $x$ & & & \\
\hline & $\begin{array}{l}4=\text { Uso de } \\
\text { software web }\end{array}$ & & & & & $\mathrm{x}$ & & \\
\hline \multirow{3}{*}{ Cognitivas } & $\begin{array}{l}5= \\
\text { lectoescritura }\end{array}$ & & $X$ & & $\mathrm{x}$ & & & \\
\hline & $\begin{array}{l}6=\text { análisis y } \\
\text { redacción de } \\
\text { texto }\end{array}$ & $x$ & & & & & & \\
\hline & $\begin{array}{l}7=\text { redacción } \\
\text { para la } \\
\text { expresión oral }\end{array}$ & & & & & & $x$ & \\
\hline \multirow{6}{*}{ Emocionales } & $\begin{array}{l}8= \\
\text { concentración } \\
\text { durante } \\
\text { periodos } \\
\text { prolongados }\end{array}$ & $x$ & $X$ & $x$ & $x$ & & & $X$ \\
\hline & $\begin{array}{l}9=\text { paciencia } \\
\text { y poder de } \\
\text { frustración }\end{array}$ & $x$ & & & & & $x$ & $\mathrm{X}$ \\
\hline & $\begin{array}{l}10=\text { voluntad y } \\
\text { responsabilidad }\end{array}$ & $x$ & & & & & & \\
\hline & $\begin{array}{l}11=\text { confianza } \\
\text { en sí misma y } \\
\text { en la estrategia }\end{array}$ & $x$ & & & & $x$ & $x$ & $x$ \\
\hline & $\begin{array}{l}12=\text { trabajo en } \\
\text { equipo }\end{array}$ & $x$ & & & & & $x$ & $x$ \\
\hline & $\begin{array}{l}13= \\
\text { intensión de } \\
\text { comunicación }\end{array}$ & & & & & & $x$ & \\
\hline
\end{tabular}

Fuente: elaboración propia. 


\begin{tabular}{|c|c|c|c|c|}
\hline \multicolumn{2}{|c|}{ Competencias } & \multirow[t]{2}{*}{ Adquiridas } & \multirow[t]{2}{*}{$\begin{array}{c}\text { A } \\
\text { rehabilitar }\end{array}$} & \multirow{2}{*}{$\begin{array}{c}\begin{array}{c}\text { A } \\
\text { desarrollar }\end{array} \\
x\end{array}$} \\
\hline \multirow{4}{*}{ Digitales } & $1=$ movimiento preciso para el uso de periféricos & & & \\
\hline & $2=$ Uso de comunicadores & & & $x$ \\
\hline & $3=$ Uso de software de ofimática & $x$ & & \\
\hline & $4=$ Uso de software web & $x$ & & \\
\hline \multirow{3}{*}{ Cognitivas } & $5=$ lectoescritura & $x$ & & \\
\hline & $6=$ análisis y redacción de texto & $x$ & & \\
\hline & $7=$ redacción para la expresión oral & & & $x$ \\
\hline \multirow{6}{*}{ Emocionales } & $8=$ concentración durante periodos prolongados & & $\mathrm{x}$ & \\
\hline & $9=$ paciencia y poder de frustración & & & $x$ \\
\hline & $10=$ voluntad y responsabilidad & $x$ & & \\
\hline & $11=$ confianza en sí misma y en la estrategia & & $x$ & \\
\hline & $12=$ trabajo en equipo & $x$ & & \\
\hline & 13= intensión de comunicación & & $x$ & \\
\hline
\end{tabular}

Fuente: elaboración propia.

En la figura 1 se presenta un esquema de las tecnologías de apoyo necesarias para la realización de las tareas. Se comienza por la estudiante, quien realiza las tareas, luego se grafican las tecnologías implicadas en el factor ergonómico para el acceso al ordenador; los periféricos, rampa digital y configuraciones de accesibilidad del sistema operativo que habilitan el ingreso de información (input) al ordenador; las tecnologías con las que esta información es procesada, y las que vehiculizan el egreso de información (output) del ordenador para la comunicación con otra persona. 
Figura 1. Tecnologías de apoyo que median la realización de las tareas

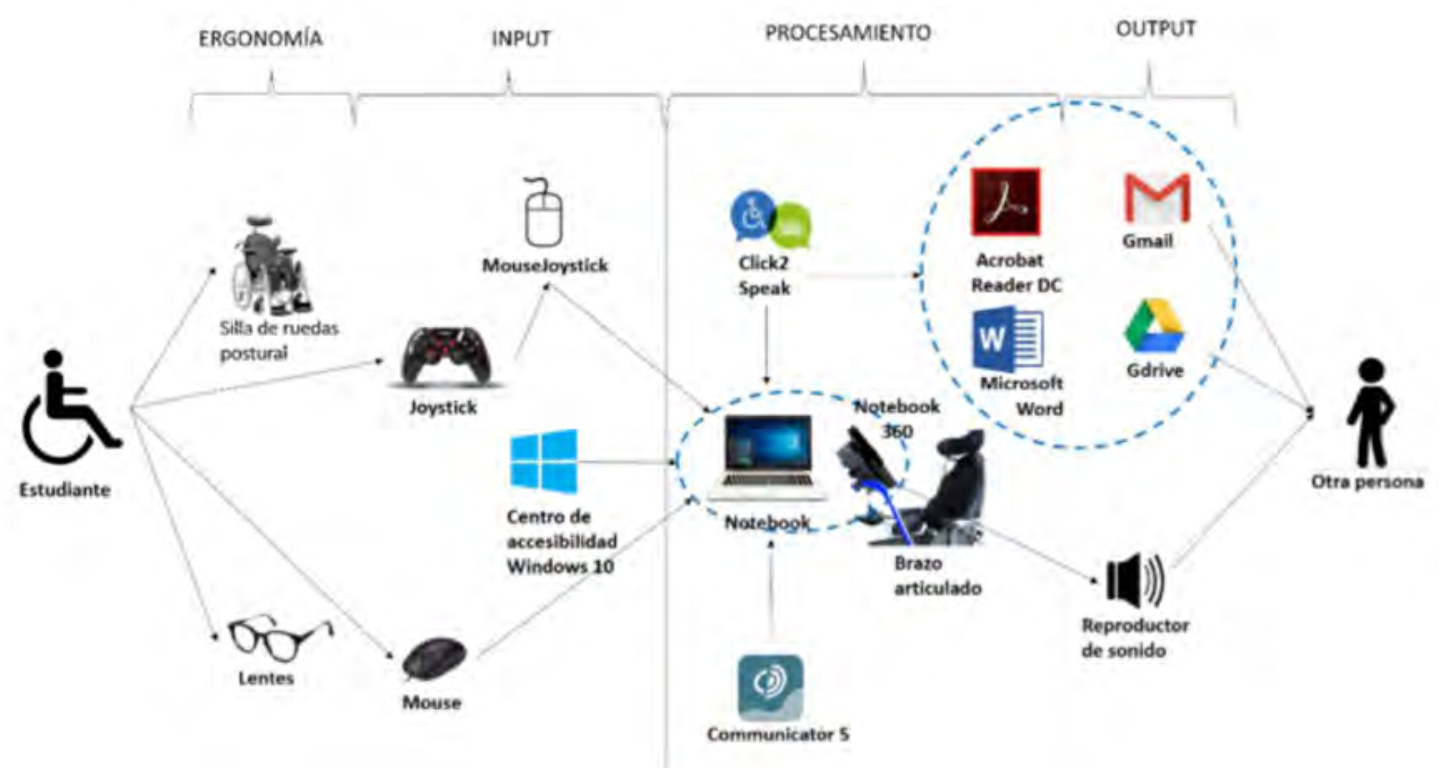

Fuente: elaboración propia.

Se emplearon las siguientes tecnologías de apoyo: silla de ruedas postural y lentes, periférico alternativo joystick adaptado y mouse adaptado, rampa digital MouseJoystick, configuración del centro de accesibilidad del sistema operativo Windows 10, dos notebook (la primera luego se reemplazó por la segunda y se le agregó un brazo articulado para sujetarla a la silla de ruedas), dos software de comunicación (Clic2Speak, que también funciona como teclado digital y control de comandos, y Communicator 5), dos software de escritorio (Acrobat Reader DC y Microsoft Word 2016) y dos online (Gmail y Gdrive).

Interpretar y cumplimentar consignas (Tarea -T- 1), emular el habla (T6) y acceder al ordenador (T7) fueron las tareas que le requirieron a Sol el ejercicio de la mayor cantidad de competencias. A su vez, la mayor parte de ellas fueron desarrolladas por primera vez en el marco de la estrategia: movimiento preciso para el uso de periféricos (joystick y mouse) (Competencia -C-1) que le permitieran acceder a la notebook mediante la rampa digital MouseJoystick; redacción para la expresión oral (C7) mediante el uso de los softwares de comunicación Click2Speack (en su función de comunicador) y Communicator 5 ; y paciencia y poder de frustración (C9) durante el proceso de aprendizaje inherente a la apropiación de las tecnologías.

Sol ya sabía utilizar los software de escritorio (C3) Acrobat Reader DC y Microsoft Word 2016, y web (C4) Gmail y Gdrive; emplearlos no implicó un aprendizaje para ella. La diferencia es que, ahora, debía hacerlo mediante periféricos (C1) alternativos y, a su vez, adaptados; y la rampa digital. Algo similar sucede con la competencia 5. Si bien el daño neurológico no afectó su lectoescritura (C5), para seguir el texto a leer en la interfaz (T2) y para escribir (T4), debió aprender a utilizar Clic2Speak en la función de teclado digital y control de comandos. 
Sol tenía adquirida y conservada la competencia cognitiva: análisis de texto (C6), y también las competencias emocionales: voluntad y responsabilidad $(C 10)$ y trabajo en equipo $(C 12)$. Ella fue tenaz durante el desarrollo de la estrategia y cooperó en todo momento en el quehacer conjunto.

En cuanto a las competencias a rehabilitar, si bien Sol antes de su receso de la actividad académica estaba acostumbrada a estudiar durante tiempos prolongados (C8), fue necesario rehabilitar esta competencia al desarrollar la estrategia. Lo mismo sucedió respecto a su autoestima, ante el cambio de sus circunstancias de vida fue necesario fortalecer la confianza en sí misma y en la estrategia (C11) que desarrollamos juntas para su inclusión educativa.

Respecto a la intención de comunicación (C13), Sol es de personalidad tímida, de poco hablar. Sumada esta característica a los años de silencio que vivió por no acceder a una tecnología de apoyo que emulara su habla, ella naturalizó "vivir sin hablar". Fue necesario estimular esta competencia, y aprender a utilizar los software de comunicación (C2).

En la figura 2 se presenta la red de personas e instituciones que se conformó en el proceso de desarrollo de la estrategia y que permitió su sustentabilidad en el tiempo.

Figura 2. Red de personas e instituciones

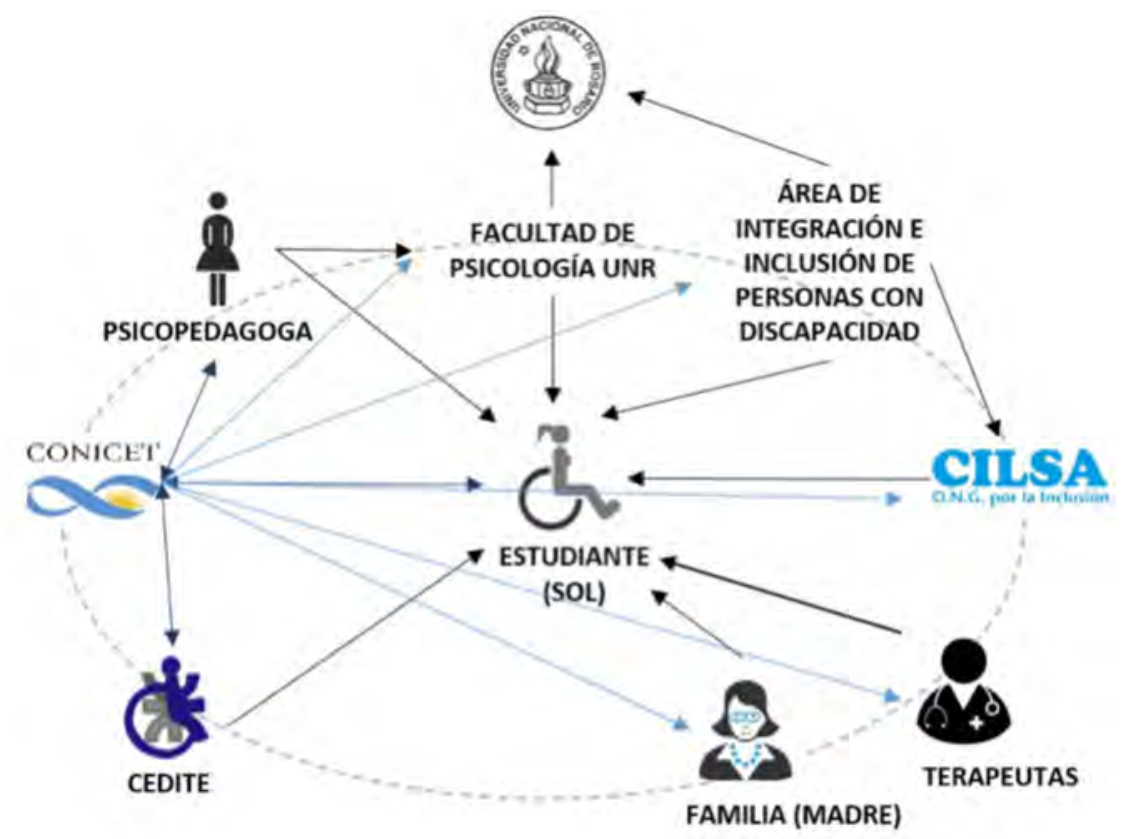

Fuente: elaboración propia.

Nota: el círculo de puntos agrupa a todos los actores sociales que intervinieron en la estrategia. Los nombro en el orden en que fueron incorporándose al desarrollo de la estrategia: el CeDITE, el CONICET, Sol, su madre y terapeutas, la facultad de Psicología de la UNR, el área de integración e inclusión de personas con discapacidad también de la UNR, la Organización No Gubernamental (ONG) CILSA, y una psicopedagoga. Sol se encuentra en el centro. Todos los actores se relacionan con ella. A su vez, algunos de ellos se relacionan entre sí. EI CONICET, institución a la que pertenece la autora de este trabajo, también se vincula con todos los actores por ser la entidad que origina la estrategia y convoca a la participación de los demás en el que se fueron incorporando para su desarrollo. 
En adelante se describe cómo tareas, competencias, tecnologías y red de personas e instituciones se vinculan en el desarrollo de la estrategia.

\subsection{Estudiante otra vez}

La alianza que dio origen al proyecto fue el convenio de colaboración entre el CeDITE y el CONICET. En este contexto tuvo lugar el primer encuentro con Sol, cuando expresó su deseo de volver a estudiar en la Facultad de Psicología de la UNR. A fin de ampliar el conocimiento respecto a sus circunstancias de vida, se contactó luego con su familia (principalmente la madre) y terapeutas, quienes colaboraron con la estrategia durante todo su desarrollo en diferentes instancias.

La UNR comenzó a participar en la estrategia cuando se reinscribió a Sol como alumna regular. Con las autoridades se realizó un balance de las asignaturas aprobadas, regularizadas y faltantes para tener conocimiento sobre su estado de avance actual; y se acordó una cantidad de adecuaciones ${ }^{2}$ al "Reglamento de alumnos regulares y libres" (resolución 081-96CD) y al "Reglamento de evaluación académica" (resolución 203-05CD) en atención a las singularidades de Sol y sus circunstancias de vida.

Ella decidió comenzar por rendir el final de una de las asignaturas ya regularizadas: Metodología de la Investigación Psicológica 1 (MIP1). Se asesoró a la docente a cargo para adaptar la modalidad de examen final. Ella seleccionó la bibliografía a estudiar. El material estaba digitalizado, pero no en un formato accesible a personas con limitaciones en la movilidad de las extremidades ${ }^{3}$, como es el caso de Sol. Se hizo accesible el material de estudio y se realizó un tutorial de manera que quedasen asentados los pasos para que en oportunidades futuras fuera la institución quien se responsabilizase de la tarea.

La profesora elaboró dos módulos de examen con preguntas de respuesta múltiple y justificación. Se acordó que Sol realizaría la evaluación con anterioridad al examen, a libro abierto (pudiendo consultar la bibliografía), usando un procesador de texto y desde su casa. Luego se presentaría al examen con el documento impreso. En ese mismo momento, la profesora lo leería, le daría una devolución oral, y completaría la libreta universitaria con la nota que correspondiera.

Este primer reto: rendir el examen final de MIP1, sirvió de motivación para la construcción de la estrategia, y de prueba para identificar las tareas a realizar y las competencias y tecnologías de apoyo necesarias.

2. Entre los puntos más relevantes se encuentran:

- Sol podrá asistir a clases con un/a acompañante o asistente.

- Las excepciones en el calendario de cursada serán publicadas en el sitio web de la facultad o comunicadas a Sol personalmente a fin de evitar traslados innecesarios.

- Se la exime de cumplir con el $75 \%$ de asistencia a clase para regularizar las asignaturas. Su asistencia y permanencia en la Facultad se ajustará a sus posibilidades y circunstancias.

- Las adaptaciones curriculares se llevarán a cabo entre el/a facilitador/a y los docentes. Estos últimos deben participar activamente en el proceso de adaptación.

- La bibliografía deberá ser entregada a Sol en formato digital accesible por el docente a cargo.

- Las materias que regularice no tendrán vencimiento. Sol será evaluada en la mesa de examen final disponible que a ella le resulte más oportuna.

- En la instancia de evaluación podrá exponer el contenido teórico haciendo uso de sus tecnologías de apoyo. El docente deberá disponer del tiempo necesario para la evaluación.

3. Para que sea accesible, el texto debe estar en una sola columna y desprotegido para su copia y cambio de formato. Documentos .doc o .pdf elaborado a base de imágenes no son accesibles. Para accesibilizar este tipo de documentos, no queda más opción que utilizar un software para el Reconocimiento Óptico de Caracteres. 
El primer desafío a resolver se vinculó al factor ergonómico, y se relaciona con la postura y visión de Sol para su acceso a la notebook.

Sobre la postura, Sol tenía una silla de ruedas deteriorada que ya no cumplía con la función de mantenerla en una posición adecuada. Estudiar durante horas en esa silla era perjudicial para su salud, por lo que la familia aceleró el trámite para obtener una nueva.

Como se anticipó en la introducción, el daño neurológico afectó significativamente la visión de Sol. Ella no podía leer por sí misma. Hacía tiempo que no consultaba a un oftalmólogo debido a que su última experiencia había sido muy frustrante: como el médico no logró entender sus respuestas, no pudo ayudarla. Se buscó otro oftalmólogo y acudió acompañada a la consulta por la autora de este trabajo, quien medió el diálogo entre Sol y el médico. Él le recetó lentes que mejoraron su visión en un $50 \%$. Ahora sí era posible que leyera por sí misma de un ordenador, pero no sin adaptaciones de la interfaz.

Sol disponía de una notebook. Para configurar la interfaz a sus requerimientos, fue necesario cambiar la versión 7 del sistema operativo Windows por una 10 que facilitó el CeDITE. Esta versión, a diferencia de la 7 , permite escalar los elementos en la interfaz a un $125 \%$ y que este cambio impacte sobre todas las aplicaciones instaladas incluyendo los menús de funciones. También se mejoró el brillo y el contraste activando el ClearType (hace que el texto en la pantalla sea más nítido), y se configuró un puntero de mayor tamaño.

Para acceder a la notebook, se probaron diferentes periféricos adaptados y alternativos, así como rampas digitales. Entre ellos, la cámara web en combinación con un software de traking head (el puntero sigue la punta de la nariz), traking eyes (el puntero sigue el iris de los ojos), y mouse trackball. Finalmente se encontró que el indicado para Sol era un joystick de comando sensible y un mouse, a los cuales se les pegó un botón para evitar que los dedos de Sol resbalasen por fuera del área en la que debe realizar el movimiento (figura 3). Ambos periféricos son posibles de conseguir en cualquier comercio de computación a un precio muy bajo. Con el dedo índice de la mano izquierda Sol utiliza el joystick; y con los dedos índice y mayor de la derecha, el mouse.

\section{Figura 3. Adaptación de joystick y mouse de bajo costo}
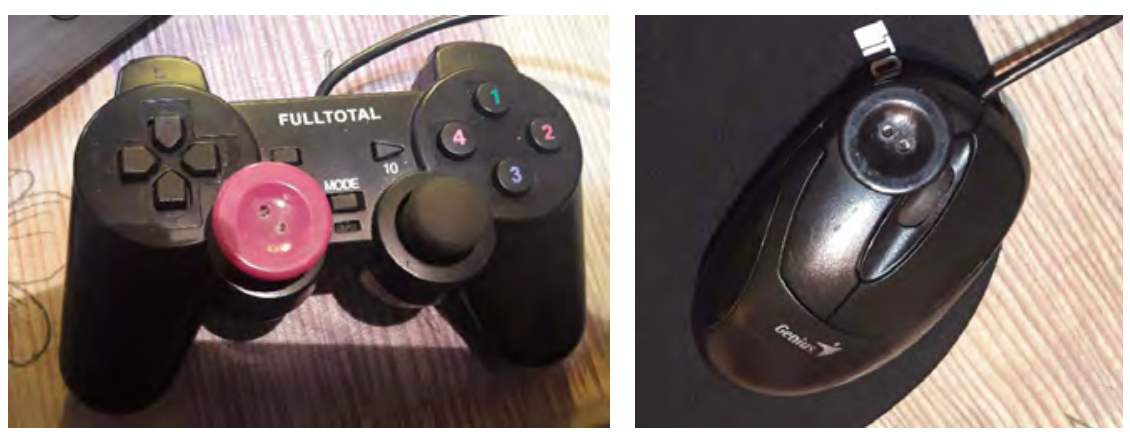

Fuente: fotografías tomadas por la autora de este trabajo. 
Para emular el cursor en la interfaz se utilizó la rampa digital de libre acceso MouseJoystick, con velocidad 5 y sin clic automático. Junto con la kinesióloga, Sol continuó practicando el uso de estos periféricos, lo que aceleró significativamente su aprendizaje para un uso eficiente.

Se abrió una cuenta de Google para Sol. Ella recibió el programa de estudio de MIP1 y la bibliografía mediante la mensajería Gmail y el repositorio on-line Gdrive, ambas herramientas de libre acceso. Con la participación de la facilitadora descargó los textos y los ordenó en carpetas en la notebook; luego leyó, marcó los textos y copió partes de ellos, para lo que usó Acrobat Reader DC de libre acceso; con las partes de los textos elaboró resúmenes en Microsoft Word 2016 también facilitado por el CeDITE.

Para el uso de Microsoft Word 2016, Acrobat Reader DC, Gmail y Gdrive se evaluaron varios teclados virtuales, entre ellos Virtual Key Board. Se decidió utilizar el software de libre acceso Click2Speak, que permite acoplar a estos sistemas un teclado virtual alfanumérico que incluye comandos para redactar, navegar en el texto, copiar y pegar entre los más empleados por la estudiante. Haciendo uso de estos sistemas elaboró los exámenes a entregar.

A diferencia del teclado en pantalla de Windows 10, Click2Speak funciona también como software de comunicación: le permite a Sol redactar mensajes y emitirlos por síntesis de voz. Esta función fue fundamental para su interacción con la facilitadora durante la realización de las tareas académicas.

\subsection{Habitar la facultad}

Sol acudió a la mesa de examen, entregó su trabajo y aprobó MIP1. Volver a ingresar a la facultad y transitar los espacios a los que hacía tiempo deseaba volver fue una experiencia trascendental que motivó la expansión de la estrategia: Sol ya no quería habitar la facultad en ocasiones aisladas, ahora deseaba asistir con mayor frecuencia. Para esto, era necesario que tuviera la posibilidad de (1) concurrir a las clases al menos una vez por semana y (2) de contar con tecnologías de apoyo que le permitieran emular el habla a fin de participar en la clase si lo deseaba o conversar con compañeros y docentes.

Respecto al primer punto, Sol precisa de un transporte especial para ir y volver de su casa a la facultad. La obra social no cubre traslados a otro lugar que no sea el centro de rehabilitación y, a su vez, su familia no contaba con recursos económicos para pagarlo de forma particular. Respecto al segundo: para emular el haba de Sol era necesario obtener tecnologías de apoyo nuevas con requerimientos específicos, lo que implicaba una inversión económica de la cual la familia de Sol no disponía.

Se realizó el pedido de un subsidio económico al Área de integración e inclusión de personas con discapacidad de la UNR para costear las tecnologías que Sol precisaba y el traslado de una vez por semana a la facultad. La UNR le otorgó una beca económica de transferencia mensual y, a su vez, generó un convenio con la ONG CILSA que terminó por completar el monto solicitado.

Con este dinero, Sol adquirió una notebook de tipo 360, su principal atributo es que puede "convertirse en tablet" sin perder las cualidades de una notebook. Con la ayuda de un herrero se adaptó un brazo articulado provisto por la UNR a fin de que en un extremo sostenga el ordenador a la altura de la mirada de la estudiante, y en el otro se ajuste a la silla de ruedas (figura 4). 
Luego de evaluar varios software de comunicación, entre ellos Plaphoons, elegimos el Communicator 5 y se instaló en la notebook 360. Aunque es de tipo privativo, característica que complejiza su adquisición, se consideró que era el más eficiente para Sol. La licencia se la regaló una persona en una situación de discapacidad similar a la de la estudiante. Para fortalecer la intención de comunicación, ejercitar la redacción de mensajes para la expresión oral y el aprendizaje del uso de Communicator 5 , se acudió a la fonoaudióloga de la estudiante a quien se capacitó en el uso de estas tecnologías.

Figura 4. Sistema portátil de comunicación

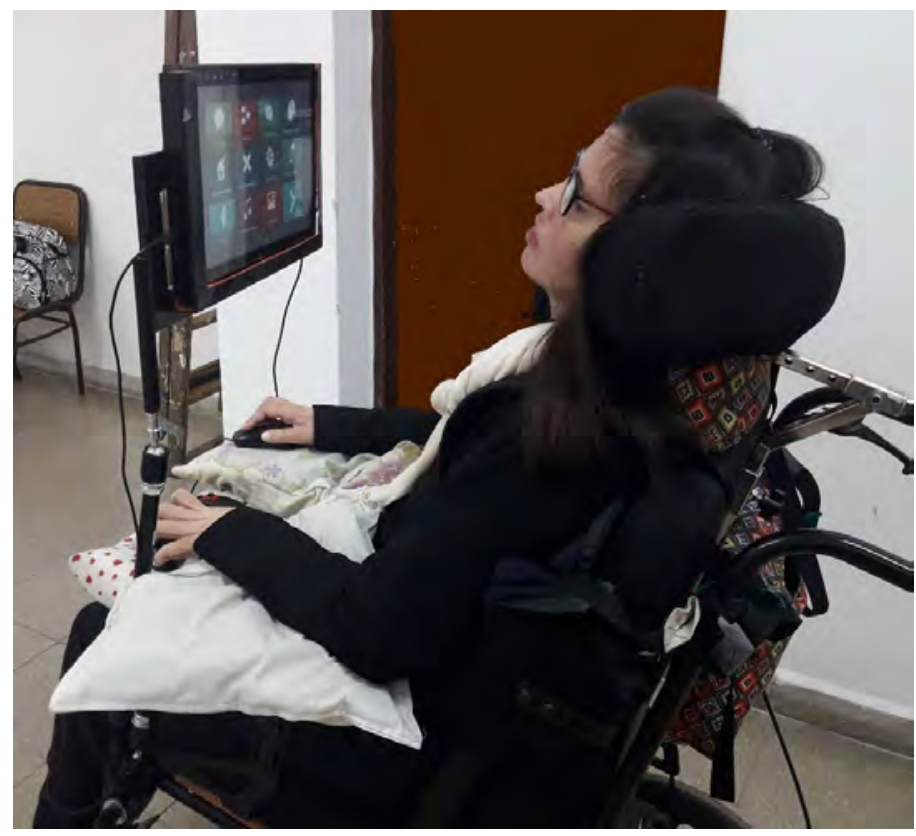

Fuente: fotografía tomada en la Facultad de Psicología de la UNR por la autora de este trabajo.

Nota: el brazo articulado sujeta la notebook 360 a la altura de la mirada de Sol. En la interfaz se ve la pantalla de inicio de Communicator 5 , en su mano izquierda el joystick y en la derecha el mouse.

\subsection{Actualidad y prospectiva}

Para la sustentabilidad de la estrategia en el tiempo, fue necesario incorporar un profesional que pudiera acompañar a Sol en su proceso de aprendizaje mediado por tecnologías de apoyo, interactuar con los docentes a cargo de las materias para trabajar con ellos en la adaptación curricular y garantizar que las pautas de accesibilidad académica fueran respetadas. Se eligió con Sol a una psicopedagoga. La obra social, no sin resistencias, finalmente otorgó su prestación.

Considerando que los terapeutas de Sol pueden cambiar, y que es necesario que los mismos cooperen con el sustento de la estrategia, se realizaron tutoriales para la descarga, configuración y uso de todas las 
tecnologías de apoyo utilizadas que fueron ubicados en una carpeta compartida con Sol en Gdrive. De esta manera los terapeutas podrán evacuar dudas o inconvenientes técnicos con la mayor autonomía posible.

Desde que Sol se reincorporó a la universidad ha aprobado cuatro materias y, actualmente, cursa una con modalidad presencial. Durante este proceso algunos docentes no respetaron las pautas de accesibilidad académica para Sol, obstaculizando su proceso de aprendizaje ${ }^{4}$. Debido a estas resistencias, junto con la psicopedagoga se procedió a elaborar un documento para formalizar dichas pautas mediante un protocolo. Se esperó el aval de la secretaría académica de la Facultad y la predisposición de la UNR para otorgarle carácter de decreto a fin de que su cumplimiento no pueda ser evitado ni cuestionado.

\section{Reflexiones finales}

Si bien establecer generalizaciones no es prudente hasta tanto se contraste este caso con otros, en lo que a esta experiencia respecta se deduce que sin la implementación de la estrategia descrita, la accesibilidad universitaria de Sol no hubiera sido posible. Aun estando en la lista de personas con discapacidad, hasta que el CONICET intervino, la UNR no generó accione para la inclusión de Sol. Las leyes por sí mismas no construyen un contexto inclusivo. La accesibilidad a la educación superior universitaria de ciudadanos argentinos con discapacidad, y especialmente con dificultades de acceso a la información y a la comunicación, sigue siendo un desafío.

¿Qué características demandó la estrategia para la accesibilidad académica de Sol?

Para que una estrategia sea genuina, es importante mantener la escucha a la persona que busca su inclusión. Pueden surgir miedos, obstáculos, momentos de mayor o menor entusiasmo, pero lo que no puede faltar es el deseo de participar, de formar parte. En el caso de Sol, fue su deseo de volver a estudiar y a la vez impotencia por no acceder a la información y a la comunicación, lo que impulsó el desarrollo de esta estrategia.

La estrategia debe construirse en el contexto de su implementación, no diseñarse previamente. Para esto es pertinente asumir desafíos que habiliten al sujeto vivenciar experiencias de referencia. En el caso de Sol, estudiar y rendir MIP1 fue la experiencia que tomamos de referencia para identificar las tareas a realizar, y las competencias y tecnologías de apoyo necesarias a este fin.

Parte de la estrategia es lograr que los diferentes actores sociales que intervienen en su desarrollo asuman el compromiso de colaborar y lo sostengan en el tiempo. De esta forma es posible generar acuerdos y alianzas en el seno de una red de relaciones en la que cooperan personas e instituciones. Es preciso que uno de los actores sociales que integran esta red funcione como nexo conector y mantenga la cohesión. Al comen-

4. Ejemplo de estas situaciones son: no responder mails ni llamadas para acordar cuestiones relativas a la bibliografía a estudiar y modalidad de evaluación, entregar a la estudiante bibliografía no accesible, y no respetar el tiempo que ella precisa en el examen final para responder haciendo uso de sus tecnologías de apoyo. 
zar la estrategia de accesibilidad académica de Sol, fue la autora de este trabajo (en el rol de facilitadora) quien desempeño este rol. Más tarde, tomó este lugar la psicopedagoga que se incorporó.

Las tecnologías de apoyo de tipo software y hardware son un medio irrenunciable para el acceso a la información y a la comunicación de personas con limitaciones motrices en las extremidades y, a su vez, ausencia de lenguaje elocutivo como es el caso de Sol. Son muchas las tecnologías de apoyo de este tipo disponibles, encontrar las adecuadas es fundamental para evitar frustraciones que fatiguen al estudiante. A su vez, no se debe olvidar que las competencias de la persona pueden cambiar con el tiempo, por lo que se debería evaluar con cierta periodicidad si las tecnologías siguen siendo adecuadas, deben reconfigurarse o suplantarse por otras.

Las estrategias para la accesibilidad académica de estudiantes con discapacidad no son transferibles, sino que deben construirse en función a las singularidades de cada caso. Las tareas a realizar por los estudiantes varían según la carrera, las competencias son diferentes en cada estudiante, las tecnologías de apoyo se eligen y configuran o desarrollan en relación a las tareas y competencias y, finalmente, las personas e instituciones que intervienen en el desarrollo de la estrategia cambian en cada contexto. Es posible identificar interrogantes, variables y/o fases que podrían tomarse de referencia o antecedente, pero es necesario situar la estrategia en el contexto de su desarrollo. Cuando se trata de personas con discapacidad, la estandarización conduce a la exclusión.

La estrategia se expande en la medida en que el estudiante supera sus metas. Inicialmente Sol no quería ir a la Facultad más que para rendir exámenes. Pero con su primera visita a la institución, emergió el deseo de ir con mayor frecuencia y, por lo tanto, la búsqueda de los medios para que puediera hacerlo. Identificar nuevas demandas y asumir nuevos desafíos constituye un ciclo reflexivo que propicia la expansión de la estrategia.

Para terminar, se destaca que la intervención fue trascendental en la calidad de vida de la estudiante. En una agenda semanal cubierta de turnos a diferentes terapeutas; se sumaron horas de estudio, mesas de examen y asistencia a la Facultad. Son actividades que no están subjetivamente atravesadas por el tratamiento del cuerpo, sino por su deseo de estudiar la carrera de Psicología. 


\section{Referencias bibliográficas}

Argentina. Acuerdo Plenario N798/11, 4 de octubre de 2011. Extensión, Documento Programa Integral de accesibilidad. Consejo Interuniversitario Nacional CIN (en línea). <http://normativa.cin.edu.ar/buscador_normativas. php?ah=st5b031300c8fb1\&ai=notascin||85000002\&id_documentos=3606\&tm=1>, acceso 22 de noviembre de 2019.

Argentina. Resolución CE 426/07, 8 de agosto de 2007, Extensión. Programa integral de accesibilidad en las universidades públicas. Consejo Interuniversitario Nacional CIN (en línea). <http://www.cin.edu.ar/doc. php?id=1130>, acceso 22 de noviembre de 2019.

Argentina. Ley 26.378, 21 de mayo de 2008, Apruébase la Convención sobre los Derechos de las Personas con Discapacidad y su protocolo facultativo, aprobados mediante resolución de la Asamblea General de las Naciones Unidas del 13 de diciembre de 2006. Infoleg (en línea). <http://servicios.infoleg.gob.ar/infoleglnternet/ anexos/140000-144999/141317/norma.htm>, acceso 22 de noviembre de 2019.

Argentina. Ley 27.044, 19 de noviembre de 2014, Otórgase jerarquía constitucional a la Convención sobre los Derechos de las Personas con Discapacidad. Infoleg (en línea). <http://servicios.infoleg.gob.ar/infoleglnternet/ anexos/235000-239999/239860/norma.htm>, acceso 22 de noviembre de 2019.

Argentina. Ley de Educación Superior 25.573, 11 de abril de 2002, Modificación de la Ley № 24.521. Infoleg (en línea). <http://servicios.infoleg.gob.ar/infoleglnternet/anexos/70000-74999/73892/norma.htm>, acceso 22 de noviembre de 2019.

Naciones Unidas (2006): Convención sobre los Derechos de las Personas con Discapacidad. Nueva York: ONU (en línea). <https://www.ohchr.org/SP/HRBodies/CRPD/Pages/disabilitiesconvention.aspx>, acceso 22 de noviembre de 2019.

Presidencia de la Nación Argentina (2018): Plan nacional de discapacidad (2017- 2022). Buenos Aires: Agencia Nacional de Discapacidad (en línea). <https://www.argentina.gob.ar/sites/default/files/plan_nacional_discapacidad_texto_04-2018.pdf>, acceso 22 de noviembre de 2019.

Stake, R. E. (1999): Introducción "Estudio intensivo de los métodos de investigación con estudio de casos", en Stake, R. E.: Investigación con estudio de casos. Madrid: Morata.

Wikinclusion (en línea). <http://wikinclusion.org/index.php/P\%C3\%A1gina_principal>, acceso 18 de junio de 2018. 